\title{
特別講演
}

\section{STRUCTURE AND FUNCTION OF von WILLEBRAND FACTOR}

\author{
Jean-Pierre GIRMA and Dominique MEYER \\ Hopital Bicêtre, Paris, France
}

von Willebrand Factor (vWF) is an adhesive, multimeric, glycoprotein present in plasma, platelets and subendothelium (SE), which has two functions : first it serves as carrier protein for Factor VIII, second, it plays a crucial role in platelet adhesion to SE as it represents a "bridge" between platelet membrane GPIb and GPIIb/IIIa and SE components like collagens and glycosaminoglycans. vWF is involved at high shear rates in the initial contact of platelets with the SE, in their subsequent spreading and in thrombus formation. The binding of $\mathrm{vWF}$ to GPIb can both induce and regulate the subsequent interaction with GPIIb/IIIa but requires prior interaction of vWF with SE components or, in vitro, the presence of agonists such as ristocetin or botrocetin. The mapping of specific fragments of $\mathrm{vWF}$ involved in platelet adhesion has been performed using a series of proteases and well-characterized monoclonal antibodies (MAbs) to distinct epitopes of the protein. Several functional domains have been identified by several groups, including ours, on the $275 \mathrm{kDa}$ subunit comprising 2050 amino acids. The study of patients and animal models and the use of compounds (aurin tricarboxylic acid, botrocetin, glycocalicin), MAbs, recombinant fragments and synthetic peptides emphasize the role of the vWF-GPIb axis in platelet-vessel wall interactions and in paricular that of sequences adjacent to or within the Cys 509 -695 disulfide loop of vWF. The size of the vWF multimers, the sequences 540-578 and 692-708 as well as the positively charged portions within the Cys 509-695 disulphide loop appear to play a key role to promote and modulate the primary interaction of vWF with platelet GPIb. 